\title{
Feature extraction to predict quality of segregating sweet tamarind using image processing
}

\author{
Panana Tangwannawit ${ }^{1}$, Sakchai Tangwannawit ${ }^{2}$ \\ ${ }^{1}$ Department of Mathematics and Computing Science, Faculty of Science and Technology, \\ Phetchabun Rajabhat University, Phetchabun, Thailand \\ ${ }^{2}$ Department of Information Technology Management, Faculty of Information Technology and Digital Innovation, \\ King Mongkut's University of Technology North Bangkok (KMUTNB), Bangkok, Thailand
}

\begin{tabular}{l} 
Article Info \\
\hline Article history: \\
Received Aug 18, \\
Revised Nov 24, 202 \\
Accepted Dec 1, 202 \\
\hline Keywords: \\
Classification \\
Feature extraction \\
Image processing \\
Sweet tamarind
\end{tabular}

\begin{abstract}
In this modern age, several new methods have been developed, especially in image processing for agriculture business, which consists of technologies derived from artificial intelligence (AI) capabilities called machine learning. Classify is a widely used method to analyze patterns, trends, as well as the body of knowledge from the data visualization. Image classification application improves discrimination and prediction efficiency. The objective of this research was to feature extraction of sweet tamarind and compare the algorithm for classification. This research used images from golden sweet tamarind species with the use of MATLAB and python language. The steps of this research consisted of 1) preprocessing step for finding the distance to appropriate of the image quality, 2) feature extracting for finding the number of black pixels and the number of white pixels, perimeter, diameter, and centroid, and 3) classifying for algorithms' comparison. The results showed that the camera's distance to the image was $60 \mathrm{~cm}$. The coefficient of determination was at 0.9956 , and the standard error of estimate was $7,424.736$ pixels. The conclusion of classification found that the random forest had the highest accuracy at $92.00 \%$, SD. $=8.06$, precision $=90.12$, recall $=92.86$, and F1-score $=91.36$.
\end{abstract}

This is an open access article under the CC BY-SA license.

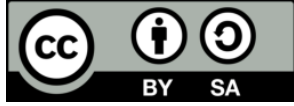

\section{Corresponding Author:}

Sakchai Tangwannawit

Department of Information Technology Management

Faculty of Information Technology and Digital Innovation

King Mongkut's University of Technology North Bangkok (KMUTNB)

Bangkok 10800, Thailand

Email: sakchai.t@itd.kmutnb.ac.th

\section{INTRODUCTION}

Artificial intelligence (AI) has played numerous important roles in the development of organizational functions. Implementation of the technologies [1] such as image processing [2], internet of things (IoT) [3], intelligent control [4] and robotics, signal processing, natural language processing (NLP) [5], and big data analytics [6]. The implementation of AI and image processing technology in the agriculture sector [7], this integration concept promotes the development of innovations, tools, and methodologies which can help smart farming more effectively.

Smart agriculture system improves efficiency and increases productivity per area using technology and artificial intelligence in agriculture. The intelligence system starts from a seed selection, soil quality, monitoring throttling light and temperature. Using AI and IoT systems can determine the proper amount of 
nutrients and water, planting management, and pest control. An innovative agriculture system enables accurate production forecasts, reducing costs and reducing the use of human labor.

In 2020, the smart agriculture technology market was worth more than $\$ 26$ billion and compound annual growth rate (CAGR). It is worths more than one-third of the market value in the Asia-Pacific region. The value of the smart agriculture market in Thailand was $\$ 128.7$ million in 2018 and is expected to reach $\$ 269.9$ million in 2022. Apparently, an intelligent algorithm like machine learning and tools [8] allows automated machines to learn and utilize the trained algorithms to solve problems for humans.

In this research, the classification of distinctive features of images was used, designed for various applications. For example, image classification algorithms are used to learn and classify data. The algorithms, such as k-nearest neighbors (KNN) [9], decision tree [10], random forest [11], support vector machine (SVM) [12], and logistic regression [13] were used. In addition, selecting the most appropriate data classification algorithm to extract the distinctive features of the images in the dataset is crucial. The algorithm will measure the models' best performance, including accuracy, precision, recall, and f1-score. This research divided data into two parts: a training set and test set by k-fold cross validation (K-fold), stratefied k-fold (SK-fold) and leave one out cross validation (LOOCV). In this study, 5-fold cross validation was combined with python language to classify data. It is also used to obtain reliable results to measure the extraction efficiency and key features of the sweet tamarind.

The results were then used to develop a standardized size sorting tool for sweet tamarind. More results can be applied in sorting the packing size of the sweet tamarind for export. The abovementioned technologies have been used in the agricultural sector to increase production efficiency, reduce the production cost, and save time and labor. Moreover, the production quality can be precisely controlled.

\section{THEORETICAL BACKGROUND AND RELATED RESEARCH}

\subsection{Image processing}

Image consists of small units called pigment or pixel in which each point has a numerical value.

There are 4 types of general images processing:

- Original images-an image formed by a combination of three primary colors which vectors are showing red, green, and blue values [14].

- Gray scale image - an image with a color in grayscale. In 8-bit, there are 256 possible colors ranging from black (0) to white (255) [15].

- Binary images-an image consisting only of black and white. There are only two values in black and white image dots: black as 0 and white as 1 or 255 [16].

- Histogram-graph which presented total numbers of dots in the image. The horizontal axis shows the intensity level from 0-255. When the grayscale is low, the intensity with less values will be seen as black. If grayscale is high, this means that the image is very intense and will be seen as white. The vertical axis of the graph shows a number of image dots valued at each intensity range.

Feature extraction for image analysis [17], feature is an analysis of the main characteristics to find representatives of data to use to represent that image [18]. This theory on image analysis is commonly used by using feature extract of objects to find specific features and used them to analyze the characteristics of the image of sweet tamarind. This enables finding the sweet tamarind standard size. The methods for representing images with specific image characteristics commonly used the properties of color, texture, shape, and histograms of oriented gradiants (HOG) [19]-[20].

\subsection{Classification algorithm}

Region, area recognition of an object, or boundary, recognition of an object's boundary, which is also known as pattern are the working approaches of shape classification [21]. There are two types of shape classification methods: decision theoretic and structural aspects [22]. Decision theoretic type recognizes quantities such as length, area, and texture [23]. The second type uses a pattern, which works best for classification. In this research, classification algorithm consists of KNN, decision tree, random forest, support vector machine (SVM) and logistic regression [13].

\subsection{Validation of method verification}

Verification is a method used to test the ability of the model that will be used as a model to predict data that needs to be classified in the future. This is done by considering the percentage and accuracy of the prediction of the data to be categorized. In this research, there are two methods for validation: k-fold cross validation (k-fold), Stratefied K-fold (SK-fold) and leave one out cross validation (LOOCV). 


\subsubsection{K-fold cross validation (K-fold), stratefied K-fold (SK-fold)}

$\mathrm{K}$-fold $\mathrm{CV}$ is a method for dividing dataset into parts. The principle is summarized as to divide the data into $\mathrm{k}$ parts [11]. One piece of data is required to test a model that has been created referred to as test set. The rest of the k-1 data is used to create the model in classification and often called training set, alternating the test until all parts are used as the test set. After that, the percentage of the correct prediction is considered which most of the training sets are divided into three-fourths of the total data. The remainder is a test set which is considered as one of the most commonly used methods for predicting the data to be classified.

\subsubsection{Leave one out cross validation (LOOCV)}

LOOCV is another method wildly accepted equally to the method one. The principle in summary is assuming that there are total of $\mathrm{N}$ data, one value will be taken out which remains $\mathrm{N}-1$ to be used to create a model in the classification of groups [13]. After data was pulled out, calculations will keep repeating in this manner until all data is correct and then used for testing [14]. After that, percentage of the correct prediction is considered.

\subsection{Sample}

Sweet tamarind is a popular fruit highly consumed in many countries across the world including Thailand. Sweet tamarind can be stored for a long time. In 2019, the volume of sweet tamarind being exported were as high as 19,902.6 tons [24]. The value of export was 440.07 million baht. Thailand's major trading partners are China, United Arab Emirates, Vietnam, the United States of America, Malaysia, Singapore, Indonesia, Saudi Arabia, Laos, and Kuwait. Most of them export around 70\% and sell around $30 \%$ domestically. The main countries that order most sweet tamarinds are China [25] and Vietnam, but they do not require high quality tamarind or grade-B products. While countries such as the United States of America, European countries, and the Middle East would only order high quality, or grade-A products.

This research investigates the feature extraction method that provides the correctness value of the dataset [26], [27] but the improve of previous research were to enhanced algorithm of classification techniques and feature extraction of texture of objects. This was done by utilizing the image processing and using data classification from the research of [9], [28]-[30] with feature extraction method and used to create criteria for sweet tamarind quality classification by image processing method. Calculating the area of an image in pixels and comparing the algorithm performance to classify data by defining the extraction interval method using sum and variance of mean number of pixels can be used to sort sweet tamarind grades and quality.

\section{RESEARCH METHOD}

150 pieces (pictures) of high quality golden sweet tamarind were used. The samples were divided into two groups depending on their sizes: 80 standard A size and 70 standard B size. A size was high quality than B size, that consists of 1) A size big than B size and 2) A size have a diameter larger than B size. The area was set for capturing the images with black background. The image of sweet tamarind must be the resolution of 10 million pixels. The step of research development consisted of preprocessing, feature extraction and evaluation as explained in Figure 1.

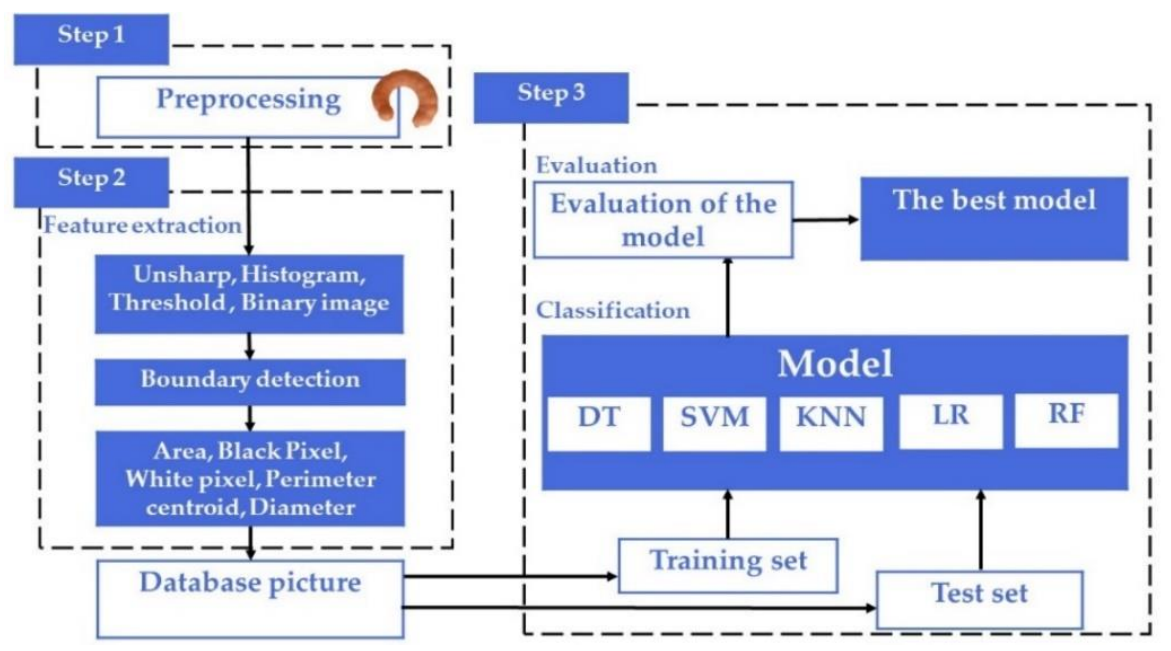

Figure 1. Framework of research method 


\subsection{Step 1: preprocessing}

The distance between the camera and the object was determined to control the image quality of the tamarind and so that the measured value from the system can be read in the best image. Therefore, calibration at different distances was needed. The distance from the reference object to camera in this study was used at 5 different levels, $20 \mathrm{~cm}, 30 \mathrm{~cm}, 40 \mathrm{~cm}, 50 \mathrm{~cm}$, and $60 \mathrm{~cm}$. The test object require paper at $7 \times 7 \mathrm{~cm}\left(49 \mathrm{~cm}^{2}\right)$, $6 \times 6\left(36 \mathrm{~cm}^{2}\right) \mathrm{cm}, 5 \times 5 \mathrm{~cm}\left(25 \mathrm{~cm}^{2}\right)$, and $4 \times 4 \mathrm{~cm}\left(16 \mathrm{~cm}^{2}\right)$ size to determine the linear correlation trend of the area at $\mathrm{R}^{2}$ of the standard area based on regression analysis [13]. The value then was calculated in (1).

$$
y_{i}=\beta_{0}+\beta_{1} x_{i 1}+\beta_{2} x_{i 2}+\cdots+\beta_{k} x_{i k}+\varepsilon_{i}, i=1, \ldots, n
$$

\subsection{Step 2: feature extraction}

Feature extraction was used starting from making unsharp mark to filter the image before processing. This is to sharpen the image of the sweet tamarind. After that, the equation to calculate the black pixel size and white pixel size of the image are used. Threshold values are obtained by determining the color histogram in the pixel points of interest to separate the background image from the image. Total pixel size of the image was identified. The size of white pixels, size of black pixels, perimeter, cenrtoid, and diameter of the object were calculated from the image using MATLAB.

- $\quad$ Step 2.1: Getting color image (RGB) of sweet tamarind and improve the image through unsharp mark method. This converts the color image of the tamarind into grayscale image with the intensity of each point in the position of the tamarind image. By reading the value obtained from the image taken in $\mathrm{X}, \mathrm{Y}$ coordinates, the pixel value was adjusted, then the obtained $\mathrm{Y}$ values are converted back to the coordinates of $\mathrm{X}, \mathrm{Y}$. After all pixel values were obtained, $\mathrm{Y}$ values is then used to be plot in graph in a form of histogram to show the brightness at different stage of the sweet tamarind and the images were capture at different appropriate point. This method was used to separate the objects from the background. It can be calculated from the number of frequencies in the histograms and counting the values and pixel points that is less than the crop level value by counting total number of pixels of that object, and then calculate the average of intensity level of the object through threshold method. Lastly, the resulting grayscale image was converted into two values: black (0) and white (1 or 255$)$ or binary image. The resulting sweet tamarind image was separated into two-pixel groups, the foreground image, and the background image.

- Step 2.2: Image segmentation separates the object from the background to reduce the complexity and change the display boundary to make it easier to analyze. Boundary detection method was used to separate the images into regions from the examination of each group of objects to find the edges of the sweet tamarind images. MATLAB program function was used.

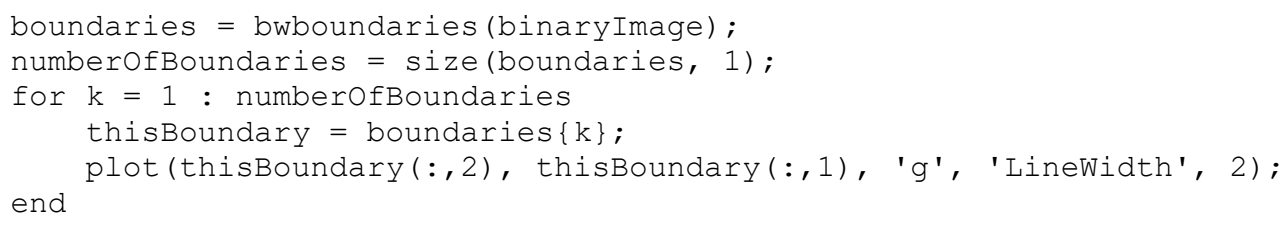

- $\quad$ Step 2.3: Table 1 shows descriptions of the attributes of image data in database to store outstanding characteristics of sweet tamarinds to store in database picture.

Table 1. Attributes of image data in database

\begin{tabular}{ccc}
\hline No & Name of data & Type of data \\
\hline 1 & Area & Double \\
2 & Black pixel & Double \\
3 & White pixel & Double \\
4 & Perimeter & Double \\
5 & Centroid & Double \\
6 & Diameter & Double \\
7 & Grade & Varchar \\
\hline
\end{tabular}

\subsection{Step 3: classification}

In this step, the extraction of dataset from tamarind image from step 2 was identified and the data was divided into two parts: training set and test set by k-fold cross validation, stratified k-fold, and loocv methods. 5-fold cross validation was used with python language for classify data. After that, four benchmarks were then used to measure the effectiveness of the tests: accuracy, precision, recall, and F1-score. 


\section{RESULTS}

\subsection{Result of camera distance measurement on image quality of sweet tamarinds}

The Table 2 shows the result of experiment to determine the distance of the camera to the image to obtain the quality of the sweet tamarind image by setting the camera distances at 5 different point: $20 \mathrm{~cm}, 30$ $\mathrm{cm}, 40 \mathrm{~cm}, 50 \mathrm{~cm}$, and $60 \mathrm{~cm}$, and using the area of the object at $49 \mathrm{~cm}^{2}, 36 \mathrm{~cm}^{2}, 25 \mathrm{~cm}^{2}$, and $16 \mathrm{~cm}^{2}$. The finding of linear equation was to plot graph with $\mathrm{x}$-axis (area of the object) and y-axis (number of pixels) for calculated with linear equation. In Figure 2, example of the camera distances at $60 \mathrm{~cm}$. In order to test the straight-line equation, it was found that the $\mathrm{R}^{2}$ value of the distance at $60 \mathrm{~cm}$ had the most significant relationship between the distance and the area of the object at 0.9956 . The standard of error in forecasting caused by the regression equation was at the value 7,424.736 pixels in which in this research, it was photographed at $60 \mathrm{~cm}$.

Table 2. Results of linear equation for length between camera and object

\begin{tabular}{cccc}
\hline \multirow{2}{*}{ Length } & \multicolumn{3}{c}{ Linear Regression } \\
& Linear Equation & $\mathrm{R}^{2}$ & Standard Error of Estimate \\
\hline 60 & $\mathrm{y}=6366.4 \mathrm{x}+6269$ & 0.9956 & $7,424.736$ \\
50 & $\mathrm{y}=9010.3 \mathrm{x}+304.14$ & 0.9574 & $33,180.84$ \\
40 & $\mathrm{y}=14071 \mathrm{x}-20927$ & 0.9829 & $32,410.54$ \\
30 & $\mathrm{y}=22949 \mathrm{x}-21099$ & 0.9952 & $27,818.88$ \\
20 & $\mathrm{y}=48104 \mathrm{x}-126554$ & 0.9900 & $84,237.99$ \\
\hline \multicolumn{2}{c}{ Coefficient of determination $\left(\mathrm{R}^{2}\right)$}
\end{tabular}

Coefficient of determination $\left(\mathrm{R}^{2}\right)$

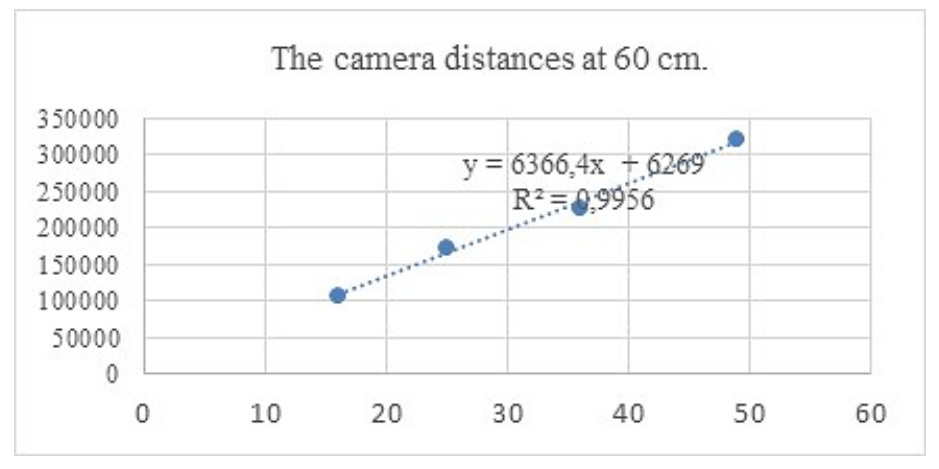

Figure 2. Shows the results from image processing

\subsection{Results from the featured extraction}

The Figure 3 shows the results obtained from MATLAB programming language for image processing are consist of: Figure 3(a) original image produced by converting the RGB image of sweet tamarind to black and white image through unsharp mask technique. Figure 3(b) histogram image generated from black and white image was used to determine the grayscale value. Figure 3(c) binary image is the conversion of grayscale value image into binary image (black and white) and Figure 3(d) boundaries image is first to determine the edges of the image. After that, it will be used to find the black pixel and white pixel to calculate the area of the image in the unit as pixel. The equation was used to calculate and find the number of black pixel and number of white pixels, perimeter, diameter, and centroid through MATLAB programming language.

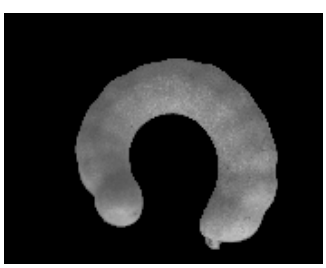

(a)

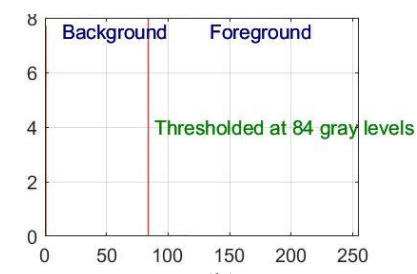

(b)

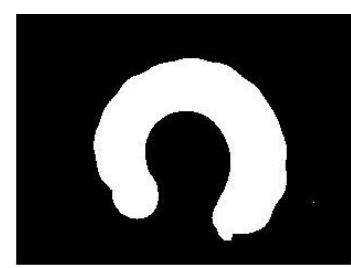

(c)

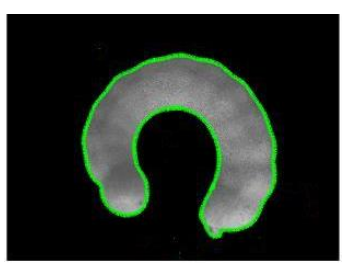

(d)

Figure 3. Results from the featured extraction steps to: (a) original image, (b) histogram image, (c) binary image and (d) boundaries image 


\subsection{Algorithm benchmark results for classification}

The data group was categorized into two parts: training set and test set with the use of k-fold crossvalidation, stratified k-fold, and loocv. In this study 5-fold cross-validation was used. Moreover, four measures were used to measure the effectiveness of the tests which were accuracy, precision, recall, and f1score. It was found that random forest had the highest accuracy. The data were then taken to find the crossvalidation score to confirm the accuracy of the data as shown in Table 3 and 4 . It was found that random forest had the highest accuracy at $92.00 \%$, sd $=8.06$, precision $=90.12$, recall $=92.86$, and $\mathrm{F} 1$ - score $=91.36$. The principle of random forest was to train the same model multiple times and multiple instances on the same data set. Each training session will select different parts of the data that are being trained. Then take the decision of those models to vote on which class is the most chosen. So, the results of the algorithm found that the random forest was better than another algorithm.

Table 3. Results of accuracy and standard deviation from 5-fold cross validation

\begin{tabular}{lcccccc}
\hline \multicolumn{1}{c}{ Algorithm } & \multicolumn{2}{c}{ K-Fold } & \multicolumn{3}{c}{ Stratified K-Fold } & \multicolumn{2}{c}{ LOOCV } \\
& Accuracy & SD & Accuracy & SD & Accuracy & SD \\
\hline Decision tree & 89.33 & 4.42 & 88.67 & 11.47 & 85.00 & 35.71 \\
Support Vector Machine (SVM) & 87.33 & 9.98 & 90.00 & 5.58 & 85.00 & 35.71 \\
K-Nearest Neighbors (KNN) & 89.33 & 12.18 & 91.33 & 4.52 & 89.00 & 31.29 \\
Logistic regression & 84.67 & 9.57 & 90.00 & 5.16 & 86.00 & 34.70 \\
Random forest & 91.33 & 3.40 & 92.00 & 8.06 & 90.00 & 30.00 \\
\hline
\end{tabular}

Table 4. Results of evaluation matrices from stratified 5-fold cross validation

\begin{tabular}{lccccc}
\hline \multicolumn{1}{c}{ Algorithm } & Accuracy & SD & Precision & Recall & F1-score \\
\hline Decision tree & 88.67 & 11.47 & 86.02 & 88.57 & 88.80 \\
Support Vector Machine (SVM) & 90.00 & 5.58 & 86.30 & 94.29 & 89.92 \\
K-Nearest Neighbors (KNN) & 91.33 & 4.52 & 88.39 & 94.29 & 90.95 \\
Logistic regression & 90.00 & 5.16 & 87.33 & 92.86 & 89.72 \\
Random forest & 92.00 & 8.06 & 90.12 & 92.86 & 91.36 \\
\hline
\end{tabular}

\section{CONCLUSION}

This research utilized image extraction feature through image analysis technique in combination with a classifier algorithm, which used algorithms with different fundamental characteristics to compare the results. In this experiment a total of 150 golden, matured, sweet tamarind species were used and divided into two groups depending on their size. 80 pieces of standard size A, 70 pieces of standard size B, and the image shooting area was prepared with black background. The results from this experiment showed that the optimal photographic distance was at $60 \mathrm{~cm}$. Then the image attributes were extracted by using MATLAB programming language to store image attribute data into a database. This database consisted of area, black pixel, white pixel, perimeter, diameter, and centroid. Then these data were then categorized into two parts which were training set and test set by k-fold cross-validation, stratified k-fold, and loocv method. Stratified 5-Fold cross-validation was also used.

After that, the model's performance was measured with accuracy value. It was found that random forest had the highest accuracy, and the mentioned data was used to find the stratified 5-Fold cross-validation to confirm the model's performance. Based on accuracy, precision, recall, and F1-score value, it was found that random forest still had the highest accuracy.

In terms of next step for this research, internet of things (IoT) will be used to work with sensor devices and connect through internet to allow the device to receive and send data to one another. Data were collected from the characteristics of the sweet tamarind placement, the movement of the tamarind, and the distance between the tamarind on the conveyor belt from the real situation. This enables the collection of data to form big data to generate further machine learning which then leads to the development of an automated system of quality sorting of sweet tamarind in Thailand for future industrial applications.

\section{ACKNOWLEDGEMENTS}

This project was financially supported by the Thailand Research Fund and Phetchabun Rajabhat University, Contract No. PCRU_2564-EC25. This project was supported by Faculty of Information Technology and Digital Innovation, KMUTNB and Faculty of Sciences and Technology, PCRU. 


\section{REFERENCES}

[1] S. Tangwannawit and P. Tangwannawit, "Artificial Intelligence Theory and Applications," in Artificial Intelligence Thailand, Petchabun : AI academic, 2020, pp. 176-180.

[2] R. Gonzalez and R. Woods, "Digital Image Processing, Harlow," United Kingdom: Pearson Education Limited, 2018, pp. 234240.

[3] P. Tangwannawit and K. Saengkrajang, "An Internet of Things Ecosystem for Planting of Coriander (Coriandrum Sativum L.)," International Journal of Electrical and Computer Engineering (IJECE), vol. 11, no. 5, pp. 4568-4576, October 2021, doi: 10.11591/ijece.v11i5.pp4568-4576.

[4] P. Tangwannawit and K. Saengkrajang, "The Development of Health Communication Support System behind," in International Conference on Education and Management Innovation (IEDRC) 2014, Hong Kong, 2014, pp. 112-124.

[5] J.-S. Chou, D.-N. Truong, and C.-F. Tsai, "Solving Regression Problems with Intelligent Machine Learner for Engineering Informatics," Mathematics, vol. 9, no. 686, pp. 1-25, March 2021, doi: 0.3390/math9060686.

[6] R. Hassan, F. Qamar, M. Kamrul Hasan, A. Mohd Aman, and A. S. Ahmed, "Internet of Things and Its Applications: A Comprehensive Survey," Symmetry, vol. 12, no. 1674, pp. 1-29, October 2020, doi: 10.3390/sym12101674.

[7] P. Tanwannawit and K. Saengkrajang, "Development of Smart Internet of Things (IoT) for Local Vegetables," in The 15th National Conference and International Conference on Applied Computer Technology and Information Systems, Thailand, 2019, pp. 134-146.

[8] D. Alaminos, J. I. Peláez, M. Belén Salas, and M. A. Fernández-Gámez, "Sovereign Debt and Currency Crises Prediction Models Using Machine Learning Techniques," Symmetry, vol. 13, no. 652, pp. 1-28, March 2021, doi: 10.3390/sym13040652.

[9] Ibrahim, M. K. Osman, N. A. M. Yuso, K. A. Ahmad, N. H. Harun, and R. A. A. Raof, "Characterization of cracking in pavement distress using image processing techniques and k-Nearest neighbour," Indonesian Journal of Electrical Engineering and Computer Science, vol. 14, no. 2, pp. 810-818, May 2019, doi: 10.11591/ijeecs.v14.i2.pp810-818. S.

[10] Kang, "K-Nearest Neighbor Learning with Graph Neural Networks," Mathematics, vol. 9, no. 820, pp. 1-12, April 2021, doi: 10.3390/math9080830.

[11] N. Ilseok, D. Hae-Wo, K. Soo-Ock, K. Su-Hyun, S. Seoleun, and L. Seung-Jae, "Machine Learning-Based Hourly FrostPrediction System Optimized for Orchards Using Automatic Weather Station and Digital Camera Image Data," atmosphere, vol. 12, no. 846, pp. 2-16, June 2021, doi: 10.3390/atmos12070846.

[12] S. Ibrahim, Z. Azuren Noor Azmy, N. N. Abu Mangshor, N. Sabri, A. F. Ahmad Firdaus, and Z. Ahmad, "Pre-trained classification of scalp conditions using image processing," Indonesian Journal of Electrical Engineering and Computer Science, vol. 20, no. 1, pp. 138-144, October 2020, doi: 10.11591/ijeecs.v20.i1.pp138-144.

[13] R. Panigrahi et al., "Performance Assessment of Supervised Classifiers for Designing Intrusion Detection Systems: A Comprehensive Review and Recommendations for Future Research," Mathematics, vol. 9, no. 690, pp. 1-32, March 2021, doi: $10.3390 /$ math9060690.

[14] N. S. Parameswaran and D. Venkataraman, "A computer vision based image processing system for depression detection among students for counseling," Indonesian Journal of Electrical Engineering and Computer Science, vol. 14, no. 1, pp. 503-512, April 2019, doi: 10.11591/ijeecs.v14.i1.pp503-512.

[15] H. A. Aliyu, M. A. Abdul Razak, and R. Sudirman, "Normal and abnormal red blood cell recognition using image processing," Indonesian Journal of Electrical Engineering and Computer Science, vol. 14, no. 1, pp. 96-100, April 2019, doi: 10.11591/ijeecs.v14.i1.pp96-100.

[16] H. K. Shihab, A. M. Salama, M. Aida, and F. N. Mohammad, "Review of local binary pattern operators in image feature extraction," Indonesian Journal of Electrical Engineering and Computer Science, vol. 19, no. 1, pp. 23-31, July 2020, doi: 10.11591/ijeecs.v19.i1.pp23-31.

[17] R. A. J. Gining et al., "Harumanis mango leaf disease recognition system using image processing technique," Indonesian Journal of Electrical Engineering and Computer Science, vol. 23, no. 1, pp. 378-38, July 2021, doi: 10.11591/ijeecs.v23.i1.pp378-386.

[18] M. T. S. Al-Kaltakchi, H. Abd Al-Raheem Taha, M. Abd Shehab, and M. A. M. Abdullah, "Comparison of feature extraction and normalization methods for speaker recognition using grid-audiovisual database," Indonesian Journal of Electrical Engineering and Computer Science, vol. 18, no. 2, pp. 782-789, May 2020, doi: 10.11591/ijeecs.v18.i2.pp782-789.

[19] T. T. Ramanathan, J. Hossen, S. Sayeed, and J. Emerson Raja, "Survey on computational intelligence based image encryption techniques," Indonesian Journal of Electrical Engineering and Computer Science, vol. 19, no. 3, pp. 1428-1435, September 2020, doi: 10.11591/ijeecs.v19.i3.pp1428-1435.

[20] K. Djunaidi, H. Bedi Agtriad, D. Kuswardani, and Y. S Purwanto, "Gray level co-occurrence matrix feature extraction and histogram in breast cancer classification with ultrasonographic imagery," Indonesian Journal of Electrical Engineering and Computer Science, vol. 22, no. 2, pp. 795-800, May 2021, doi: 10.11591/ijeecs.v22.i2.pp795-800.

[21] G. Jayagopi and S. Pushpa, "Arrhythmia Classification Based on Combined Chaotic and Statistical Feature Extraction," Indonesian Journal of Electrical Engineering and Computer Science, vol. 12, no. 1, pp. 127-136, October 2018, doi: 10.11591/ijeecs.v12.i1.pp127-136.

[22] U. W. Wasekar and R. K. Bathla, "A review on supervised learning methodologies for detection of exudates in diabetic retinopathy," Journal of Advanced Review on Scientific, vol. 23, no. 2, pp. 837-846, August 2021, doi: 10.11591/ijeecs.v23.i2.pp837-846.

[23] R.-I. Chang, C.-L. Ting, S.-Y. Wu, and P.-Y. Yin, "Context-Dependent Object Proposal and Recognition," Symmetry, vol. 12, no. 1619, pp. 2-20, September 2020, doi: 10.3390/SYM12101619.

[24] Office of the Permanent Secretary Ministry of Commerce, "Situation of important agricultural products and trends 2562 ," 20 January 2019. [Online]. Available: www.ops.moc.go.th. [Accessed 2019].

[25] D. a. t. C. G. i. Guangzhou, "Sweet tamarind," in Thailand, Bangkok Thailand: Fruit, 2018, pp. 83-84.

[26] Z. Yan, "Deep indicator for fine-grained classification of banana's ripening stages," EURASIP Journal on Image and Video Processing, vol. 46, pp. 1-10, June 2018, doi: 10.1186/s13640-018-0284-8.

[27] H. F. Kareem, M. S. AL-Husieny, F. Y. Mohsen, E. A. Khalil, and Z. S. Hassan, "Evaluation of SVM performance in the detection of lung cancer in marked CT scan dataset," Indonesian Journal of Electrical Engineering and Computer Science, vol. 21, no. 3, pp. 1731-1738, March 2021, doi: 10.11591/ijeecs.v21.i3.pp1731-1738.

[28] H. Sabry et al., "Image processing based foot plantar pressure distribution analysis and modeling," Indonesian Journal of Electrical Engineering and Computer Science, vol. 17, no. 2, pp. 594-605, February 2020, doi: 10.11591/ijeecs.v17.i2.pp594-605.

[29] A. Mohammed and H. Thary Khamees, "Categorizing and measurement satellite image processing of fire in the forest greece using remote sensing," Indonesian Journal of Electrical Engineering and Computer Science, vol. 21, no. 2, pp. 846-853, 2021, doi: 10.11591/ijeecs.v21.i2.pp846-853. 
[30] A. Poad, Farhana, S. O. Noor, R. Y. Atan, J. F. Jusoh, and M. A. Hussin, "Automated detection of vehicles license plate using image processing techniques," Indonesian Journal of Electrical Engineering and Computer Science, vol. 18, no. 3, pp. 1408-1415, June 2020, doi: 10.11591/ijeecs.v18.i3.pp1408-1415.

\section{BIOGRAPHIES OF AUTHORS}

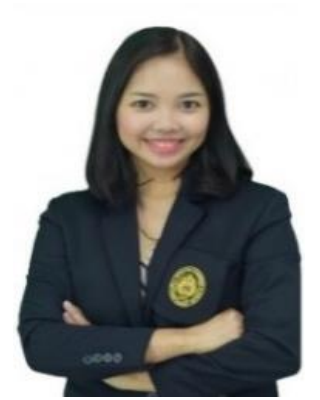

Panana Tangwannawit (iD 8 SC $\mathrm{P}$ received the professional education with B.S. (Computer Science), M.S.(Information Technology), and Ph.D. (Information Technology) from King Mongkut's University of Technology North Bangkok (KMUTNB), Bangkok, Thailand. She has been working as a lecturer for more than 24 years in the field of Computer and Information Technology in the Faculty of Sciences and Technology, Phetchabun Rajabhat University, Thailand. She can be contacted at email: panana.t@gmail.com

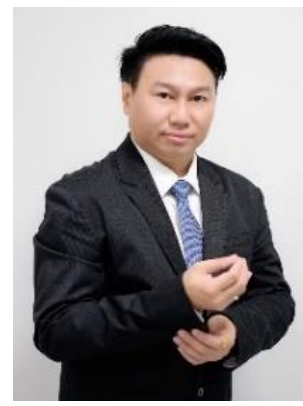

Sakchai Tangwannawit (D) SC $\mathrm{S}$ received the professional education with B.S. (Computer Science), M.S.(Information Technology), and Ph.D. (Computer Education) from King Mongkut's University of Technology North Bangkok (KMUTNB), Bangkok, Thailand. He has been working as a lecturer for morethan 10 years in the field of Information Technology in the Faculty of Information Technology and Digital Innovation, KMUTNB. He can be contacted at email: sakchai.t@itd.kmutnb.ac.th 